\title{
Population PK/PD Analysis
}

\author{
D. Concordet \\ Ecole Nationale Vétérinaire de Toulouse, Toulouse, France \\ F. Léger \\ Université Paul-Sabatier and Institut Claudius-Regaud, Toulouse, France
}

C. Ané

Ecole Nationale Vétérinaire de Toulouse, Toulouse, France

\section{INTRODUCTION}

Pharmacokinetics and pharmacodynamics are divisions of pharmacology that study the action of the body on the drug and the action of the drug on the body, respectively. They complete dose titration studies aimed to select rational dosage regimens.

The classical pharmacokinetic (PK)/pharmacodynamic (PD) studies are experimental. They are usually performed on healthy volunteers or highly selected patients to minimize interindividual variability. These studies allow to demonstrate a mechanism and to obtain rough quantitative information on the link between the dose and the effect (PK/PD behavior) of the drug.

Population PK/PD studies quantify the effect of the drug on a population of patients who could use the drug. They allow quantifying, explaining, and predicting how the variability of the drug plasma concentration acts on the variability of the drug effect. They enable optimization (individualization) of dosage regimen. Usually performed on a sample of patients who are representative of the target population, they can be considered as observational studies.

Population PK/PD relies on the use of models. A model can be defined as a simplified description of reality. Most models used in population PK/PD are statistical models. These models describe observations (concentrations and effects), but may also give some outlines of the underlying biological process. This article focuses mainly on the modelling aspects of PK/PD analysis.

First, we recall the definitions of pharmacokinetic, pharmacodynamic, PK/PD, and the population approach. The modelling approach is illustrated with the study of the curious toxic effect of topotecan, an anticancer drug. Simulations are then performed, by using estimates of the population parameters, to propose a dosage regimen with a controlled toxicity.

\section{DEFINITIONS}

One of the primary goals of drug development is to generate data that permit to select the appropriate starting dose of the drug and, subsequently, to adjust dosage to the needs of a particular patient or group of patients. Ideally, such knowledge should allow to increase the likelihood of achieving the intended therapeutic effect while reducing the risk of adverse events. One clinical development approach used to achieve this is multidose-level clinical parallel dose-response testing, which is represented in Fig. 1.

However, as advocated by Toutain, ${ }^{[1]}$ this approach does not allow provision of information on the shape of effect for a particular patient. The different doses are compared with statistical tests; thus the selected doses depend heavily on the minimum difference that can be evidenced with a given power. In this case, a large interindividual variation makes difficult the discrimination between dose effects and is considered as a difficulty in the dose selection.

A PK/PD approach gives a better insight in understanding how the body acts on the dose to give concentrations (PK step) and how variations of these concentrations are involved in the effect (PD step) (cf. Fig. 2). Holford and Sheiner ${ }^{[2,3]}$ give more precise definitions of pharmacokinetics, pharmacodynamics, and PK/PD.

\section{Population Pharmacokinetics}

Pharmacokinetic models describe the interaction between drug input and the drug disposition. Usual functions used to describe the drug input are intravenous bolus, infusion for a given time, first-order process that approximate the oral absorption.

Compartmental models are the most common approach used to describe drug disposition. Compartments are 


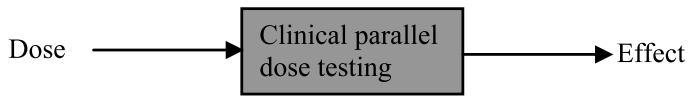

Fig. 1 A clinical design allows to study the effect of doses. It can be considered as a black box. The dose is the input; the effect is the output.

virtual spaces within which drug distribution is assumed to be uniform. A compartment is characterized by its apparent volume (which is simply the constant of proportionality between the quantity of drug it contains at a given time and the drug concentration at this time) and clearance parameters (which measure the magnitude of the rates of flow of distribution and elimination). In multicompartment models, the clearances between two compartments are assumed to be equal in both directions.

As an example, in the PK/PD analysis presented hereafter, an anticancer drug (topotecan) was administrated to $(N=42)$ women by 30 -min intravenous infusions on 5 consecutive days. Total topotecan plasma levels were analyzed according to a two-compartment model with linear elimination from the central compartment (cf. Fig. 3). The pharmacokinetic parameters were the clearance $(\mathrm{Cl})$ of the central compartment, the volume $\left(V_{\mathrm{c}}\right)$ of the central compartment, the volume $\left(V_{\mathrm{p}}\right)$ of the peripheral compartment, and the clearance $Q$ between compartments.

The drug concentrations measured in two patients receiving the same dose at the same time after a drug administration are not equal. This variability can be decomposed into two parts: a within-patient variability (essentially explained by analytical error) and a betweenpatients variability, explained by different pharmacokinetic parameters.

Population pharmacokinetics studies the variability of PK parameters and the variability of concentration profiles. The main idea, which governs population pharmacokinetics, is that each patient is characterized by one's own PK parameters. Making inference on the "population" of PK parameters is then equivalent to making inferences on the population of patients.

Patients involved in population PK studies are representative of a population of patients who intend to use the drug. The "representativeness" is measured with respect to all potential sources of variations of the drug concentrations. As an example, if the concentration

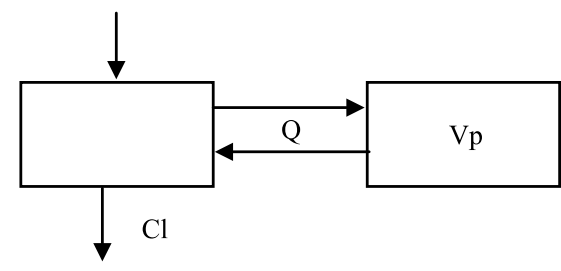

Fig. 3 A two-compartment model with linear elimination from the central compartment was used to describe the drug kinetics.

profiles observable in males are the same as the ones observable in females, it is not necessary to include both genders in the sample. Using a representative sample makes possible the identification of covariates that explain (or at least are correlated to) the concentration variability. For a given patient, it is then possible to predict (with the knowledge of its covariates) a typical concentration profile that represents the more probable profile that can be observed on patients with the same value of covariates.

The nonlinear mixed-effects models are usually used to describe these different stages of variability. They can be written as:

$\left\{\begin{aligned} C_{i j} & =f\left(\phi_{i}, t_{i j}\right)+g\left(\phi_{i}, t_{i j}, \beta\right) \varepsilon_{i j}, \quad j=1, \ldots, n_{i} \\ \phi_{i} & =A_{i} \mu+\eta_{i}, \quad i=1, \ldots, N\end{aligned}\right.$

where $C_{i j}$ is the concentration measured on the $i$ th patient at time $t_{i j}, \phi_{i}$ is the $p$-vector of individual kinetics parameters for the ith patient, $f(\phi, t)$ is the mean concentration at time $t$ for an individual with a kinetic parameter $\phi$, and $\varepsilon_{i j}$ are independent $N(0,1)$ random variables. The function $g(\phi, t, \beta)$ is the standard deviation of the error of a concentration measurement at time $t$. Usually, the different concentrations are assumed to be measured with a constant coefficient of variation $\beta$, in other terms $g(\phi, t, \beta)=\beta f(\phi, t)$.

The mean variations between the $\phi_{i}$ can be explained by covariates whose values are contained in the columns of the matrices $A_{i}$. The independent random variables $\eta_{i}$ represent the differences between the actual individual kinetic parameters and their average $A_{i} \mu$. They are assumed to be distributed according to a $N(0, \Omega)$ distribution. Thus the parameters that have to be estimated are $(\mu, \Omega, \beta)$.

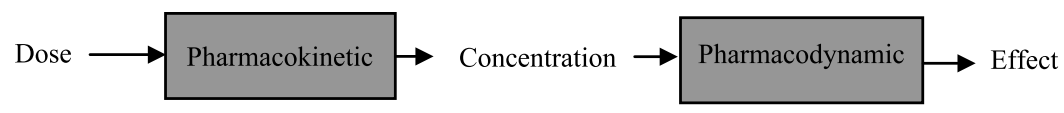

Fig. 2 The PK/PD approach splits the black box of clinical trial into two "grey boxes." The first box transforms a dose into a concentration (PK), whereas the second box links the concentration to the effect (PD). 


\section{PK/PD}

Pharmacodynamics allows to relate the drug effect (PD effect) to the concentration at its site of action; therefore, it is independent of time. The rationale for PD studies is a better understanding of the physiological response to a drug. The choice of PD response is often difficult and is out of the scope of this paper. Most drugs circulate in different tissues, but the observation of concentration at the effect site is even difficult or impossible. Actually, the drug concentrations are easily accessible; thus they are measured in plasma. For this reason, modeling mainly focuses on establishing a link between the plasma (or blood) drug concentration and the PD effect. To avoid confusion with pharmacodynamy, Holford and Sheiner suggested to name PK/PD the link between the drug plasma concentration and the PD effect.

Several classes of PK/PD models exist. The simplest ones are direct models in which there is no delay between a change in plasma drug concentration and a change in effects. This occurs when the site of action has reached equilibrium with plasma.

Indirect models allow describing a delay. This delay may be explained by the fact that the concentration is measured in plasma that is not in equilibrium with the site of action. A plot of effects-vs.-plasma concentrations (ordered by time) showing a curve (hysteresis curve) that turns counterclockwise measures the degree of lag between the change in plasma concentration and that at the effect site. A usual way to model this delay is to add an effect compartment to the kinetic model. This compartment, which is linked to the plasma concentration compartment, mimics the site of action of the drug. Because this site has usually negligible volume compared to the volume of the plasma, the PK/PD analysis is performed in two steps. First, the PK models without the effect compartment are used to estimate the PK parameters. Then PD data are analyzed with the PK model with the effect compartment in which the PK parameters obtained in the first step are maintained fixed. In this second step, only the volume of the effect compartment and its rates of exchange with the central compartment of the PK models are estimated.

The last class of PK/PD models is the physiological class. In these models, the effect of the drug is mediated by some indirect mechanism that is located separately from its site of action. The example given below is an illustration of a model of this class. An anticancer drug administered to patients gives toxicity-a decrease of neutrophil cell count. Each dividing cell is likely to be killed when this drug is present. From a macroscopical point of view, the bone marrow produces progenitor stem cells that divide rapidly, and so can be killed. If these cells survive, they continue to mature without obstacle in the bone marrow. Finally, they migrate into the blood-the observed pool—as white blood cells.

In a regulatory point of view, knowledge of PK/PD relationships can contribute to the strength of evidence to support efficacy and to address issues and questions related to the safety of drug doses and dosing regimens in certain populations of patients. The ICH E4 guideline for industry, "Dose-Response Information to Support Drug Registration," [4] describes the purpose of exposureresponse information and the uses of dose-response and/ or concentration-response data in choosing doses during the drug development process. A recent US Food and Drug Administration (FDA) guidance for industry, "Exposure-Response Relationships: Study Design, Data Analysis, and Regulatory Applications," ${ }^{[5]}$ provides recommendations for sponsors and applicants on the use of exposure-response information in the development of drugs by focusing on human studies.

\section{Population PK/PD}

If there exists a between-subjects variability in plasma drug concentration, this variability is generally lower than the one observable in effects. This is essentially due to the variety of potential sources of variations of the PD response. Population PK/PD models allow the description of this variability. These models are obtained by adding to the population PK models (Eq. 1) two components describing how the effects vary with concentration (and eventually time) and how the parameters involved in the first component vary between individuals or/and within individuals (interoccasion variability), respectively:

$$
\left\{\begin{array}{l}
Y_{i j}=N\left(\psi_{i}, \phi_{i}, t_{i j}\right)+h\left(\psi_{i}, \phi_{i}, t_{i j}, \beta\right) \varepsilon_{i j}, \quad j=1, \ldots, n_{i} \\
\psi_{i}=m+\eta_{i}, \quad i=1, \ldots, N
\end{array}\right.
$$

where $Y_{i j}$ is the response measured on the $i$ th patient at time $t_{i j}, \psi_{i}$ is the $p$-vector of individual PD parameters for the $i$ th patient, $N(\psi, \phi, t)$ is the mean PD response at time $t$ for an individual with a PK parameter $\phi$ and a PD parameter $\psi$, and $\varepsilon_{i j}$ are independent standard Gaussian random variables. The function $h(\psi, \phi, t, \beta)$ is the standard deviation of the error of a PD response at time $t$. Usually, the different responses are assumed measured with $a_{a}^{50}$ constant coefficient of variation $\beta$, in other terms $h(\psi, \phi, t, \beta)=\beta N(\psi, \phi, t)$.

As with the PK model, covariates that explain the between-subjects variability can be incorporated within the $\psi_{i}$ population mean $m$. The independent random variables $\eta_{i}$ represent the differences between the actua individual PD parameters and their average $m$. They are assumed to be distributed according to a $N(0, D)$ distribution. The parameters that have to be estimated ${ }^{\circ}$ 
are $(m, D, \beta)$. The framework of analysis for standard direct and indirect models is fully developed in Refs. [2] and [3].

Simulations that use estimates of PK and PD population parameters are performed to predict the variability of effects that can be encountered within the whole population with a specific dosage regimen. Such a simulation approach is now used to optimize the design of clinical trials.

Finally, the dosage regimen can be individualized to control the effect on a patient by taking into account the different covariates involved in the explanation of PK/ PD variability.

There is no explicit regulatory requirement regarding "population PK/PD," but the US FDA guidance for industry on population pharmacokinetics ${ }^{[6]}$ opens the door to widespread application of mixed-effects predictive models and provides guidelines that can be used for population PK/PD analyses.

\section{Software for Population PK/PD Analysis}

One of the most commonly used softwares for population $\mathrm{PK}$ and PK/PD analysis is NONMEM (the acronym for nonlinear mixed-effects modelling). ${ }^{[7]}$ NONMEM allows to fit nonlinear mixed-effects (statistical regression-type) models. A powerful feature of mixed-effects modelling is its ability to accommodate patient data as they arise in the course of routine clinical therapy, where data are typically sparse and obtained at unstructured times. The maximum likelihood estimator of the unknown parameters of models (Eqs. 1 and 2) cannot be easily computed in a closed way. The main estimation method used in NONMEM is first order (FO). It consists in approximating the model described by Eq. 1 (Eq. 2, respectively) by performing a first-order Taylor expansion of $f$ and $g$ ( $N$ and $h$, respectively) about the mean value of the random variables $\phi_{i}\left(\psi_{i}\right.$, respectively). Thus the obtained models are linear with respect to the random effects, and their parameters can be estimated using standard methods.

\section{AN EXAMPLE}

The following study gives an example of the modelization process and it shows conclusions that can be expected from a population PK/PD study. The method and results presented here are fully developed in Ref. [8].

A study was performed to find an admissible regimen, which is effective and controls the toxic effect of topotecan. Topotecan is an anticancer drug given by intravenous infusion to women with ovarian cancer as second-line therapy (cf. Ref. [9], for instance). The major toxic effect of topotecan is a decrease in neutrophil count, which occurs 8-15 days after drug administration (Fig. 4).

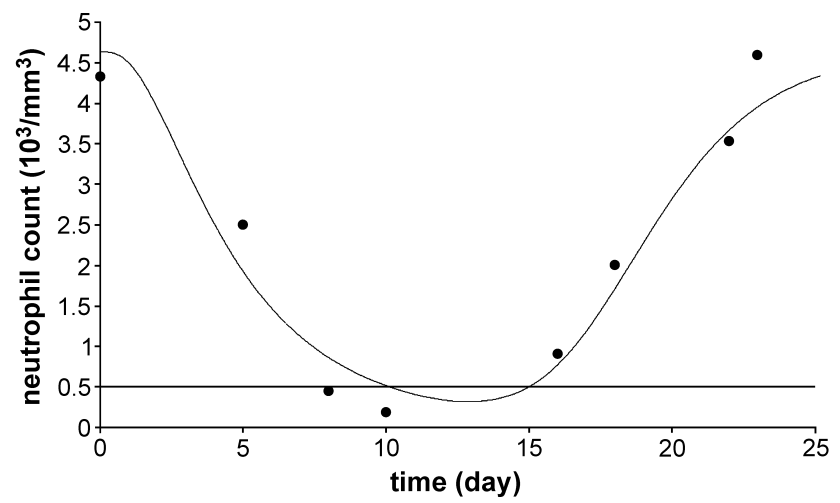

Fig. 4 An example of observed and fitted neutrophil profiles. The horizontal line at $0.5 \times 10^{3} \mathrm{PN} / \mathrm{mm}^{3}$ allows one to define the primary index of toxicity (time spent below).

A primary index used to measure this is the time the neutrophil count remains below the fixed limit: 500 PN/ $\mathrm{mm}^{3}$. Another index is the minimum neutrophil count reached. It has been shown in mice ${ }^{[10]}$ that this toxicity varies not only with the dose given, but also importantly with the duration of the exposure to the drug. Thus, when the total dose is given in a single infusion, a low toxicity is observed; but when the same total dose is fractionated over 5 days, toxicity is high. Surprisingly, when it is fractionated over 20 days or more, toxicity is reduced.

The analysis is presented in three sections. "The Pharmacokinetic Model" describes briefly the PK model and gives the population estimates. The analysis of a physiological (PD) model that explains the toxicity observed in mice and the discussion on the PK/PD link are presented in "The Pharmacodynamic Model." Then, using this model, the occurrence and magnitude of the toxicity in-patients are predicted using simulations.

\section{The Pharmacokinetic Model}

Topotecan was administrated by 30-min intravenous infusions to $N=42$ women on 5 consecutive days. For each patient, the three first daily doses were fixed a priori, and the two last doses were adjusted to reach a total area under the curve (AUC) within a targeted range $(37,500-$ $75,000 \mathrm{nM}$ min). The PK data were analyzed by Friberg et al. ${ }^{[11]}$ on a subsample of $N_{1}=31$ women. Let us recall briefly their results. Total topotecan plasma levels were analyzed according to a two-compartment model with linear elimination from the central compartment. The resulting individual parameters were the clearance $\mathrm{Cl}$ of the central compartment, the volume $V_{\mathrm{c}}$ of the central compartment, the volume $V_{\mathrm{p}}$ of the peripheral compartment, and the clearance $Q$ between compartments. This clearance $Q$ was assumed to be fixed in the population, 
Table 1 PK population parameter estimates

\begin{tabular}{lccc}
\hline & $\ln \mathbf{C l}$ & $\ln \boldsymbol{V}_{\mathbf{c}}$ & $\ln \boldsymbol{V}_{\mathbf{p}}$ \\
\hline$\hat{\mu}$ & 2.99 & 3.66 & 3.35 \\
$\sqrt{\hat{\Omega}}$ & 0.42 & 0.55 & 0.38 \\
\hline
\end{tabular}

Clearance $(\mathrm{Cl})$ is expressed in liters per hour; volumes $V_{\mathrm{c}}$ and $V_{\mathrm{p}}$ are expressed in liters.

and estimated by $\hat{Q}=46.61 \mathrm{hr}^{-1}$. The individual $\mathrm{PK}$ parameters $\phi_{i}=\left(\ln \mathrm{Cl}_{\mathrm{i}} ; \ln V_{\mathrm{ci}} ; \ln V_{\mathrm{pi}}\right)$ were assumed to be independent identically distributed (i.i.d.) and drawn from a Gaussian $N(\mu, \Omega)$ distribution, and the covariance matrix $\Omega$ was assumed to be diagonal. The estimation of $(\mu, \Omega)$ obtained from Ref. [11] in the subsample of size 31 is given in Table 1.

Finally, the individual parameters $\phi_{i}$ were predicted by the Bayesian estimates (maximum a posteriori) for all 42 patients. In the rest of this article, the kinetic profiles are considered as known and fixed.

\section{The Pharmacodynamic Model}

The discussion concerning the pharmacodynamic model is divided into three parts. First, a physiologically based structural model is chosen. Then, we consider the properties and choice of the function describing the drug action on cells. Finally, a nonlinear mixed-effects model is set up, and its parameters are estimated.

\section{The choice of the structural model}

Topotecan is a drug that acts during the replication of DNA. It binds to topoisomerase I when this enzyme is unwinding DNA. At this stage, replication is stopped, DNA is broken, and the cell dies. Thus, each dividing cell is likely to be killed when topotecan is present. From a macroscopical point of view, the bone marrow produces progenitor stem cells that divide rapidly, and so can be killed. If these cells survive, they continue to mature without obstacle in the bone marrow. Finally, they migrate into the blood-the observed pool—as white blood cells. When no drug is given, this system is at equilibrium, and can be described using the family of compartmental models given in Fig. 5.

Bone marrow constitutes a nonobserved part of the life of these cells. To get information about this part, a large number of different outputs (neutrophil profiles), obtained with a large number of different inputs (kinetics), are necessary. We have at our disposal roughly a single shape of PK profile. Remember that all the women received five consecutive daily infusions, and that the two last doses were adjusted so as to reach a target AUC. Consequently, the data are not rich enough to allow a precise description of the actual model. Therefore, we deliberately chose to use a model built on numerous data for 5-fluorouracil in rats. ${ }^{[12]}$ As this drug acts at the same stage as topotecan, it appears reasonable to take the structure of the rat model and to scale it to humans, although we will see that slight modifications are necessary. The model used in Ref. [12] contains five compartments.

Two compartments are sensitive to the drug, two are nonsensitive, and the last compartment is the blood pool. The exchanges are second-order exchanges, except for what leaves the blood, which is of order 1. All bone marrow compartments share the same second-order constant $k$. Recall that when the exchanges are of order 2 , the behavior of a given cell depends on the size of the compartment. The more cells present in this compartment, the more rapidly this particular cell will move. This type of exchange roughly mimics the birth of new cells in the compartment, and describes adequately the observed neutrophil profiles, which quickly leave and come back to baseline. In the original model ${ }^{[12]}$ the production rate was assumed to be constant, up to a feedback mechanism. More precisely, if $N_{\text {base }}$ is the baseline neutrophil count, and $N(t)$ is the neutrophil count at time $t$, then the production rate is set to $k_{\text {in }} N_{\text {base }} / N(t)$. Finally, the action of the drug is modeled by a first-order killing rate on each of the two sensitive compartments. This killing rate is assumed to be linked directly to the drug concentration $C_{t}$ at time $t: F\left(C_{t}\right)=k_{\mathrm{e}} C_{t}$.

Applying this model to data on women, the individual curve fitting was not satisfactory, for two reasons. The feedback leads to a rebound, which is pronounced in rats. As it is missing in human patients, this feedback mechanism is inappropriate for our study. Without

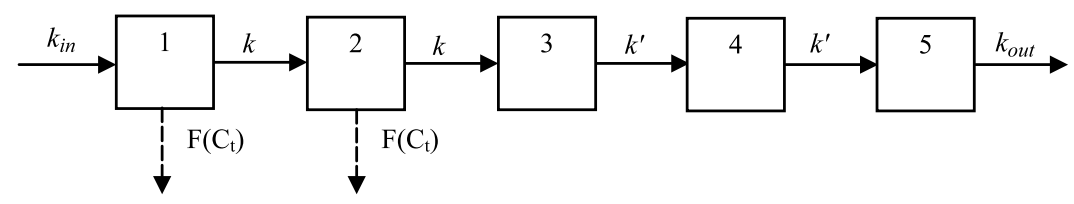

Fig. 5 General compartment model. The first four compartments are the different stages of a cell life in the bone marrow. The last䓂 compartment is the blood pool. In the original model, it was assumed that $k=k^{\prime}$ and that a feedback mechanism (a link between compartments 5 and 1) was used. 
feedback, the model used in Ref. [12] appears to be too constrained to allow a rapid decrease or increase of the curve. In this model, cells have to spend the same time in the two different states (sensitive/nonsensitive). For this reason, we chose two different rates of exchange ( $k$ and $k^{\prime}$ ), as shown in Fig. 5. The mean residence time of a cell in the nonsensitive region is then approximately proportional to the number of nonsensitive compartments, $2 k^{\prime-1}$. Similarly, the mean residence time of a cell in the sensitive region is proportional to $2 k^{-1}$ : In other respects, we notice that increasing the number of nonsensitive compartments does not determine another dynamic behavior because an increase in $k^{\prime}$ can compensate. The same argument applies to the number of sensitive compartments and the constant $k$.

In summary, there are two differences between the previous model in Ref. [12] and ours: feedback is discarded and two rates of exchange are used instead of one. The differential equations driving the selected model are now detailed.

The neutrophil profile in the blood is the solution $N(t)$, $t \geq 0$, of the system:

$$
\left\{\begin{array}{l}
\frac{\partial X_{1}}{\partial t}=k_{\text {in }}-k X_{1}^{2}-F\left(c_{t}\right) X_{1} \\
\frac{\partial X_{2}}{\partial t}=k X_{1}^{2}-k X_{2}^{2}-F\left(c_{t}\right) X_{2} \\
\frac{\partial X_{3}}{\partial t}=k X_{2}^{2}-k^{\prime} X_{3}^{2} \\
\frac{\partial X_{4}}{\partial t}=k^{\prime} X_{3}^{2}-k^{\prime} X_{4}^{2} \\
\frac{\partial N}{\partial t}=k^{\prime} X_{4}^{2}-k_{\text {out }} N
\end{array}\right.
$$

When $t=0$, the system is at equilibrium, i.e., $X_{1}(0)=$ $X_{2}(0)=\sqrt{k_{\text {in }} / k}, X_{3}(0)=X_{4}(0)=\sqrt{k_{\text {in }} / k^{\prime}}$, and $N(0)=$ $k_{\text {in }} / k_{\text {out }}$. In the following, the neutrophil profile denoted by $N(t)$ is also denoted by $N(F)(t)$ to emphasize the dependence on $F$. In the final model, $F\left(C_{t}\right)$ is set to $k_{\mathrm{e}} C_{t}$. "Drug Action: The PK/PD Link" deals with the rationale of this choice.

\section{Drug Action: The PK/PD Link}

The choice of the drug action, modeled by the killing function $F$, has not yet been discussed. The killing function $F(c)$ has some obvious properties: It cancels at $c=0$ (no drug, no action), and it is increasing (more drug, greater effect). Intuitively, the shape of $F$ determines the drug action: when $F$ is convex, low concentrations give little toxicity; but when $F$ is concave, low concentrations rapidly give toxicity. Consequently, for a fixed total dose, it seems that when $F$ is convex, a low target toxicity can be reached with a large number of small doses; whereas when $F$ is concave, the same target toxicity will be reached with a small number of high doses. Thus the shape of $F$ seems to be of major importance, especially in the context of this paper.

Actually, the impact of the shape of $F$ is reduced by the existence of a limit of toxicity. This limit is intrinsic to this family of catenary models. It is reached when the sensitive compartments are emptied (by the drug action). In that case, whatever the killing rate, the drug cannot kill more cells than those arriving in these compartments. Even if the killing rate $F\left(C_{t}\right)$ is very high, its effect on the system is very similar to the one that would be obtained with a smaller killing rate. Two different mechanisms drive the toxicity: when $F\left(C_{t}\right)$ is low (below the limit of toxicity), the shape of $F$ determines the toxicity; when $F\left(C_{t}\right)$ is high, only the time it spends above the limit of toxicity governs the effect. These intuitive considerations need to be formalized to quantify the maximal effects.

To this end, let $\Delta$ be a fixed length of time and $0<\varepsilon<1$.

Let $T=1 \sqrt{k_{\text {in }} / k}\left(T^{\prime}=1 / \sqrt{k_{\text {in }} / k^{\prime}}\right.$, respectively) be the mean residence time in the sensitive compartments (nonsensitive compartments, respectively) at steady state. Let $N_{\mathrm{b}}=k_{\text {in }} / k_{\text {out }}$ be the neutrophil count at steady state (neutrophil count at baseline). Set:

$K_{0}=\max \left\{\frac{1}{\Delta} \ln \left(\frac{20}{\varepsilon} \sqrt{\frac{k^{\prime}}{k}}\right) ; \frac{4}{3 \varepsilon \min \left\{T, T^{\prime}\right\}}\left(6+4 \frac{\Delta}{T^{\prime}}\right)\right\}$

For all killing rate functions $F\left(C_{t}\right)$, the following property is true: if $F\left(C_{t}\right) \geq K_{0}$ on an interval of length $\Delta$, say $\left[t_{0}, t_{0}+\Delta\right]$, then decreasing $F$ to $K_{0}$ on the interval $\left[t_{0}, t_{0}+\Delta\right]$ does not change the effect more than $\varepsilon N_{\mathrm{b}}$.

More precisely: $\sup _{t \geq 0}\left|N(F)(t)-N\left(F^{\left(K_{0}\right)}\right)(t)\right| \leq \varepsilon N_{\mathrm{b}}$, where $F^{\left(K_{0}\right)}=F$ outside $\left[t_{0}, t_{0}+\Delta\right]$ and $F^{\left(K_{0}\right)}=K_{0}$ on $\left[t_{0}, t_{0}+\Delta\right]$.

The proof of this result is deliberately omitted because it is too long and without practical interest. Notice that $K_{0}$, as previously defined, does not depend on $F$. The main consequence of this property is that all shapes of $F$ above $K_{0}$ give nearly the same toxicity. A simple way to explain this property is to consider the following example. Let us take the first patient of this study. Assume that her kinetics $\left(C_{t}\right)_{t \geq 0}$, as well as her structural parameters $\left(k_{\text {in }}, k, k^{\prime}, k_{\text {out }}\right)$ are known. Assume now that the killing function $F$ is a step function: $F^{(K)}(c)=0$ if $c<c_{0}, F^{(K)}(c)=K$ elsewhere, and $c_{0}$ is known. Because $\left(C_{t}\right)_{t \geq 0}$ and $c_{0}$ are fixed, the lapse of time $\Delta$ during which $C_{t} \geq c_{0}$ is fixed (e.g., $\Delta=5 \times 1$ hr). The proposition says that for all $\varepsilon<1$, there exists $K_{0}$ such that for all $K \geq K_{0},\left\|N\left(F^{(K)}\right)-N\left(F^{(\infty)}\right)\right\|_{\infty} \leq \varepsilon N_{\mathrm{b}}$.

This last inequality means, first, that toxicity is limited by the one given by $N\left(F^{(\infty)}\right)$. Second, it implies that when 
$K$ becomes large, the shape of the neutrophil curve becomes constant.

A first consequence of this property is an estimability problem of $F$. Even if the map $K \rightarrow N\left(F^{(K)}\right)$ remains injective, its derivative tends to zero (uniformly on time) when $K$ is large. Thus, if the actual $K$ of the considered individual is large (more than $K_{0}$ ), the Fisher information matrix is nearly degenerate, which implies that the maximum likelihood estimator of such a $K$ has too a large variance to be useful in practice.

Whatever is the shape of $F$ above $K_{0}$, it cannot be properly estimated with a reasonable variance. Thus whatever is the chosen parameterization for $F$, there exists an area of this parameter space where the estimation is difficult. The estimation quality (variance of the estimator) of the parameters governing the shape of $F$ below $K_{0}$ depends on the quantity of information available below this limit. If this information is poor, a large variance of the estimator is expected whatever is the parameterization of $F$. In such a case, the simplest shape for $F$ is to be preferred (i.e., a linear shape). As already mentioned, all women of the study have very similar kinetic profiles, so that the information of the data only concerns a narrow range of concentrations.

Thus we are confronted with two possibilities: either the drug concentrations lead to killing rates above $K_{0}$, thus we have no information about the shape of $F$ for small concentrations, or the drug concentrations lead to small killing rates; but because the range of these concentrations is narrow, the shape of $F$ can be documented for only a small interval of concentrations. In both cases, the linear killing rate $F(c)=k_{\mathrm{e}} c$ has to be chosen.

The second consequence drawn from this property is a qualitative explanation of the toxic behavior observed in mice. ${ }^{[10]}$ Recall that with a single dose, the observed toxicity is low: because concentrations reach high values, they lead to high killing rates (above $K_{0}$ ), but only for a short time. When this dose is fractionated over 5 days, concentrations are lower but are high enough to lead to killing rates above $K_{0}$. Because the total time spent by the killing rate above $K_{0}$ is long, toxicity is high. Finally, when the total dose is fractionated over 20 days, concentrations are too low to give high killing rates and high toxicity. Thus, our model implies a high correlation between toxicity and the time spent by the killing rate above $K_{0}$. That is exactly what is observed in Ref. [10]: a high correlation between toxicity and the time the drug concentration remains above $0.7 \mu \mathrm{M}$.

\section{The Population PK/PD Analysis}

As it can be seen on a patient-by-patient analysis, both the response curves and the individual PD parameter $\psi=$ (ln $k_{\text {in }}, \ln k, \ln k^{\prime}, \ln k_{\mathrm{e}}$ ) vary widely. The PD analysis
Table 2 PD population parameter estimates

\begin{tabular}{lcccc}
\hline & $\ln \boldsymbol{k}_{\text {in }}$ & $\ln \boldsymbol{k}$ & $\ln \boldsymbol{k}^{\prime}$ & $\ln \boldsymbol{k}_{\mathbf{e}}$ \\
\hline$m$ & -1.02 & -10.1 & -7.92 & 1.55 \\
& $\left(7.4 \times 10^{-3}\right)$ & $(0.14)$ & $(0.30)$ & $(0.076)$ \\
$\sqrt{D}$ & 0.39 & 2.07 & 0.62 & 0.84 \\
& $(0.98)$ & $(0.82)$ & $(0.23)$ & $(0.37)$ \\
$\sigma^{2}$ & 0.37 & & & \\
& $(0.17)$ & & & \\
& & & & \\
\hline
\end{tabular}

relies on the concentration time course. Recall that the individual PK profiles are known. We assume here, as have others, ${ }^{[12,13]}$ that $k_{\text {out }}$ does not vary among patients and is equal to $\sim 0.1 \mathrm{hr}^{-1}$.

The family of nonlinear mixed-effects PD models described by Eq. 2 has been used with $h\left(\psi_{i}, \phi, t_{i j}, \sigma\right)=$ $\sigma N\left(\psi_{i}, \phi, t_{i j}\right)$ and $N(\psi, \phi, t)$, with the neutrophil count defined by Eq. 3. For each patient, the neutrophil count $N\left(\psi_{i}, \phi_{i}, t_{i j}\right)$ depends on the PK parameter $\left(\phi_{i}\right)$. The $\psi_{i}$ are assumed to be independent of the PK individual parameters $\phi_{i}$. Because all the parameters $\left(k_{\mathrm{in}}, k, k^{\prime}, k_{\mathrm{e}}\right)$ are positive, we parameterized the model with their logarithm and assumed these logarithms to be distributed according to a normal distribution with mean $m$ and variance $D$.

With regard to the number of patients involved in the trial, we chose to take $D$ as a diagonal matrix. The estimation method used is FOCE; ${ }^{[7]}$ it provides an asymptotically Gaussian estimator $\hat{\theta}=\left(\hat{\sigma}^{2}, \hat{m}, \hat{D}\right)$ of $\theta$ whose asymptotic variance will be denoted by $V(\theta)$.

Table 2 gives the estimation of $\sigma^{2}, m$, and $\sqrt{D}$, as well as their asymptotic standard error (in parentheses).

It turns out that the optimized criterion (FOCE) has a large number of local minima, reflecting a large distance from the asymptotic framework. Therefore the asymptotic variance-covariance matrix of the estimator should be interpreted with care.

An example of an individual fitted curve is given in Fig. 4.

\section{Simulations and Toxicity Predictions}

This section is devoted to giving a whole set of dosage regimens with controlled toxicity. Recall that the primarye measure of toxicity is the time spent with the neutrophile counts below $500 \mathrm{PN} / \mathrm{mm}^{3}$. When this time is longer than 7 days, toxicity is considered intolerable. First, we give am index that allows one to decide qualitatively whether, for $a^{\vec{c}}$ given regimen, the toxic behavior of the drug is rather concentration-dependent (toxicity is linked to the daily dose) or time-dependent (toxicity is linked to the time spent by the drug concentration above a limit). Finally, 
we give quantitative results that determine set dosage regimens with acceptable toxicity.

\section{Simulation of a PK toxicity index}

The limit of toxicity $K_{0}$ gives information on exposure to the drug. It can be used as a surrogate that gives some useful complementary information on the toxic behavior. Indeed, as previously seen, when drug concentrations are low, the drug toxicity is concentration-dependent. But as soon as the limit $K_{0}$ is reached, toxicity depends on the time spent above $K_{0}$ and is rather time-dependent.

Let us define a toxicity index (TI). For a chosen duration $\Delta$ and a chosen $\varepsilon>0$ (these choices are discussed later), we set TI $=K_{0} / k_{\mathrm{e}}$. The main advantage of this index is that it is homogeneous to a drug plasma concentration and can be compared directly to the kinetic profile. Because both $K_{0}$ and $k_{\mathrm{e}}$ vary among patients, it is possible to simulate their distribution and then to derive the 5\% percentile of TI. In other respects, as has been shown, ${ }^{[14]}$ it is possible to use covariates such as creatinine clearance to predict for each patient the PK profile for a dosage regimen chosen a priori. As an example, assume that the expected concentration remains below the $5 \%$ quantile of TI. It means that the early sensitive compartments are not emptied by the action of the drug, with a probability of $95 \%$. This implies that the toxicity is concentrationdependent with this dosage regimen. It would not be so if the concentration time course had crossed the index TI for longer than $\Delta$.

Let us detail the rationale for the choice of $\varepsilon$ and $\Delta$. As can be seen on Eq. 4, $K_{0}$ depends on maturation times $T$ and $T^{\prime}$, whose values are about 260 and $90 \mathrm{hr}$. Therefore, $K_{0}$ is about $(120 \varepsilon)^{-1}(6+0.044 \Delta)$. Moreover, it is natural to set $\Delta$ below $24 \mathrm{hr}$ - the delay between two consecutive infusions. So the influence of the term $0.044 \Delta$ is low compared to 6 . We set $\Delta=5 \mathrm{hr}$. It remains to choose $\varepsilon$, which is a proportion of the baseline neutrophil count. If the difference between two neutrophil profiles is of the same order as the critical threshold, then the resulting toxicities are similar. Recall that the critical threshold $\left(500 \mathrm{PN} / \mathrm{mm}^{3}\right)$ is around 10 times less than the baseline neutrophil count. These considerations lead us to choose $\varepsilon=0.05$ (5\%). Even if this rough method does not tell us directly whether or not an intolerable toxicity is reached, it gives qualitative information about the toxic behavior.

\section{Simulation of admissible dosage regimens}

The aim of this part is to predict the set of admissible dosage regimen. A dosage regimen is said to be admissible if an intolerable toxicity occurs for less than a fixed percentage of patients (say 5\%). We limited ourselves to dosage regimens with constant daily doses (one infusion each day) and proceeded as follows. For a fixed dosage regimen (i.e., with a fixed number of infusions and a fixed daily dose), we determined the percentage of patients with intolerable toxicity by using a simulation method detailed hereafter. Then, the dosage regimen was adjusted to obtain a percentage equal to $5 \%$. Now, let us detail the simulation method. First, a sample $\theta_{j}^{*}=\left(\sigma_{j}^{2 *}, m_{j}^{*}, D_{j}^{*}\right)$ of size 200 was drawn from a $N(\hat{\theta}, V(\hat{\theta}))$. Next, for each $\theta_{j}^{*}$, two samples $\left(\psi_{i, j}^{*}\right)_{i=1, \ldots, 150}$ and $\left(\phi_{i, j}^{*}\right)_{i=1, \ldots, 150}$ were drawn from $N\left(m_{j}^{*}, D_{j}^{*}\right)$ and from the PK population distribution $N(\hat{\mu}, \hat{\Omega})$, respectively, given in section devoted to "Population Pharmacokinetics."

Then, $Y_{i j}^{*}(t)=N^{*}\left(\psi_{i, j}^{*}, \phi_{i, j}^{*}, t\right)$ was computed, as well as the time $T_{i j}^{*}=\int_{0}^{\infty} 1_{Y_{i j}^{*}(t) \leq 500 \mathrm{PN} / \mathrm{mm}^{3}} \mathrm{~d} t$. The proportion of patients with intolerable toxicity was then estimated as the percentage $\frac{1}{200 \times 150} \sum_{i j} 1_{T_{i j}^{*} \leq 7 \text { days }}$.

Figure 6 gives the critical daily dose obtained with these simulations as a function of the number of infusions. This figure shows that the toxicity occurrence increases with the total dose. Moreover, total doses that give few toxicities do not depend on the number of infusions: it is about $0.17 \mathrm{mg}$ for $5 \%$ toxicity and about $1.22 \mathrm{mg}$ for $10 \%$ toxicity. In other words, if the target total dose is low, the number of infusions can be chosen as desired without any change of the toxicity occurrence. A striking fact shown in this figure is that the total admissible dose giving $30 \%$ toxicity decreases from $6.2 \mathrm{mg}$ (for one infusion) to $4.9 \mathrm{mg}$ (for 15 infusions), and then it increases up to $5.1 \mathrm{mg}$ for 21 infusions. Because $6.2 \mathrm{mg}$ for one infusion produces the same toxic effect as $4.9 \mathrm{mg}$ for 15 infusions, the 1-day schedule can be considered as less toxic than the 15-day schedule (it takes more drug to produce this level of toxicity). Similarly, the 21-day schedule appears to be less toxic than the 15-day schedule. These assertions have

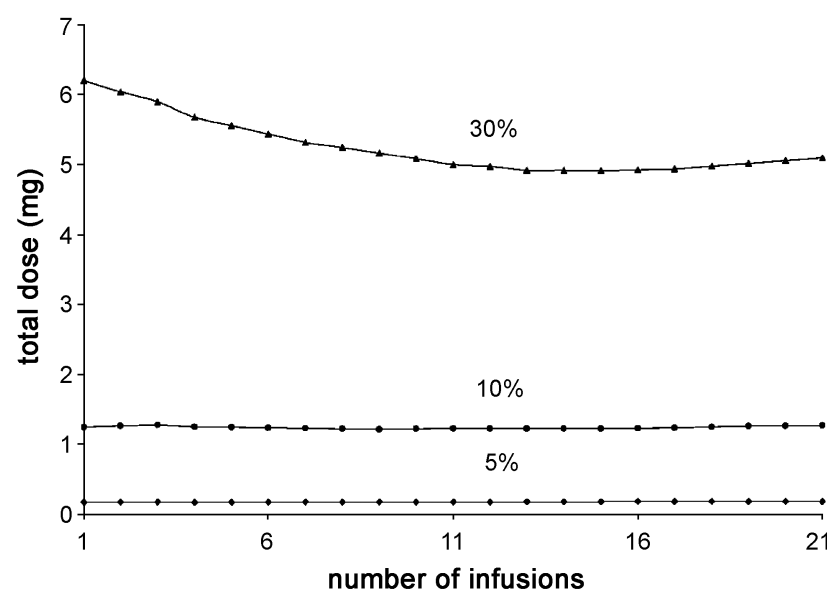

Fig. 6 Curves giving the set of dosage regimen with the same fixed occurrence of toxicity $(5 \%, 10 \%$, and 30\%). (View this art in color at www.dekker.com.) 


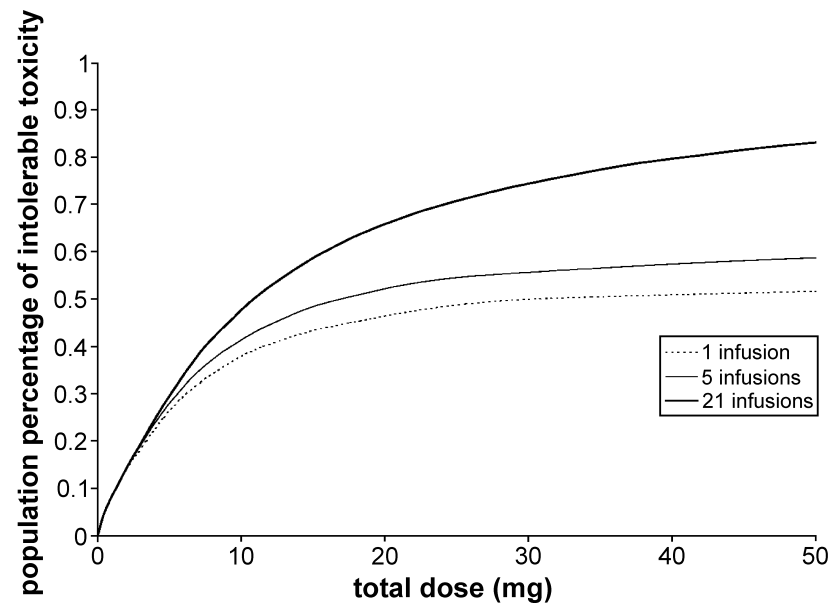

Fig. 7 Cumulative distribution function of intolerable toxicity for the 1-day, 5-day, and 21-day schedules.

been clinically evidenced (cf. Ref. [15] for instance). Figure 6 is a tool for comparing schedules in a rather qualitative way. Figure 7 gives the full quantitative description of toxic effects for thee schedules: 1,5 , and 21 infusions.

Notice that the three curves are superposed for low doses/low toxicities. This means that when the total dose is low, the occurrence of toxicity does not depend on the number of infusions, illustrating the concentration dependence and toxic behavior of the drug. When the total dose increases, the occurrence of toxicity tends to plateau at a level that depends on the number of infusions, and below $100 \%$. This is a consequence of the phenomenon described in "Drug Action: The PK/PD Link." When the limit of toxicity $K_{0}$ is reached (with high concentrations), the drug toxic behavior becomes time-dependent. Of course, this study deals only with neutropenia, and many other types of toxicity may occur with high drug doses.

Recall that five infusions were administrated and the daily dose could vary during the cycle. The total dose varied between 4.77 and $14.3 \mathrm{mg}$. As the total dose was not equally fractionated on the 5 days, we chose to simulate the percentage of intolerable toxicity in our sample as follows. For the $i$ th patient, a sample $\left(\psi_{i j}^{*}\right)_{j=1, \ldots, 100}$ was drawn from the PD population distribution $N(\hat{m}, \hat{D})$. These 100 individuals were given the PK parameters of the $i$ th patient, as well as her five daily doses. The critical times

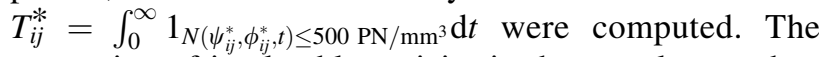
proportion of intolerable toxicity in the sample was then estimated as the percentage $\frac{1}{42 \times 100} \sum_{i j} 1_{T_{i j}^{*} \leq 7 \text { days. }}$. We ob-
tained $39 \%$.

To see if this prediction agrees with the data, the empirical percentage of toxicity in the sample was evaluated by interpolating linearly the observed neutrophil counts for each woman. With this method, only seven women $(17 \%)$ showed an intolerable toxicity, which is far from 39\%. Actually, the empirical percentage of toxicity depends on the chosen method of interpolation. As the neutrophil profiles are convex around their minimum, the linear interpolation underestimates the time spent below $500 \mathrm{PN} / \mathrm{mm}^{3}$, especially when certain observation times are far from each other. With a smooth interpolation, 19 women (45\%) showed an intolerable toxicity, which is in agreement with the predicted percentage of toxicity.

\section{CONCLUSION}

Population PK/PD modeling provides a powerful approach in the selection of adequate dosage regimen. Even if the modelization given in this paper is only the first step of a longer modelization process (range of concentrations are too narrow to allow estimation of $F$; efficacy is not studied), it shows the richness of conclusions that can be reached: the control of the effects (toxicity, efficacy, and so on) via a rational choice of individualized dosage regimen. This example also evidences the weakness of this approach.

The main impediment to the systematic implementation of this approach is the lack of methods to validate the model. Actually, the choice of a specific PK/PD model lies on a number of assumptions that are not easy to check: representativeness of the sample, normality of random effects, assumptions on the structure of variance, and so on. These assumptions have an influence on the predictions that can be drawn from a specific study (especially those obtained by simulations). Also, even when the adopted model provides a good fitting, there is no guarantee that the obtained predictions can be trusted. General statistical methods, such as tests on the variance components, would be extremely useful in the rational choice of model.

Semiparametric (nonparametric, respectively) models are probably a partial answer to some of these limitations. In these models, the distribution of the individual effects (the intraindividual random variable, respectively) is estimated. However, the properties of this family of models (at least the asymptotic ones) are not known. More work is needed in this direction.

\section{REFERENCES}

1. Toutain, P.L. Pharmacokinetic/pharmacodynamic integration in drug development and dosage-regimen optimisation for veterinary medicine. AAPS PharmSci 2002, 4 (4). Article 38 . 
2. Holford, N.H.G.; Sheiner, L.B. Understanding the doseeffect relationship: Clinical application of pharmacokineticpharmacodynamic models. Clin. Pharmacokinet. 1981, 6, 429-453.

3. Holford, N.H.G.; Sheiner, L.B. Kinetics of pharmacologic response. Pharmacol. Ther. 1982, 16, 143-166.

4. International Conference of Harmonisation E4. In DoseResponse Information to Support Drug Registration; Food and Drug Administration, Center for Drug Evaluation (CDER), November 1994.

5. Guidance for Industry. In Exposure-Response Relationships-Study Design, Data Analysis, and Regulatory Applications; U.S. Department of Health and Human Services, Food and Drug Administration, Center for Drug Evaluation (CDER), April 1999.

6. Guidance for Industry. In Population Pharmacokinetics; U.S. Department of Health and Human Services, Food and Drug Administration, Center for Drug Evaluation (CDER), February 1999.

7. Beal, S.L.; Sheiner, L.B. NONMEM User's Guide. In Nonlinear Mixed Effects Models for Repeated Measures Data; University of California: San Francisco, 1992.

8. Ané, C.; Concordet, D. Population pharmacokinetics/ pharmacodynamics relationships of an anticancer drug. Stat. Med. 2003, 22, 833-846.

9. Rowinsky, E.K.; Grochow, L.B.; Hendricks, C.B.; Ettinger, D.S.; Forastiere, A.A.; Hurowitz, L.A.; McGuire,
W.P.; Sartorius, S.E.; Lubejko, B.G.; Kaufmann, S.H.; Donhower, R.C. Phase I and pharmacologic study of topotecan: A novel topoisomerase I inhibitor. J. Clin. Oncol. 1992, 10, 647-656.

10. Guichard, S.; Montazeri, A.; Chatelut, E.; Hennebelle, I.; Bugat, R.; Canal, P. Schedule-dependent activity of topotecan in OVCAR-3 ovarian carcinoma xenograft: Pharmacokinetic and pharmacodynamic evaluation. Clin. Cancer Res. 2001, 7, 3222-3228.

11. Friberg, L.E.; Brindley, C.J.; Karlsson, M.O.; Devlin, A.J. Models of schedule dependent haematological toxicity of 2'-deoxy-2'-methylidenecytinine (DMDC). Eur. J. Clin. Pharmacol. 2000, 56, 567-574.

12. Friberg, L.E.; Freijs, A.; Sandstrom, M.; Karlsson, M.O. Semiphysiological model for the time course of leukocytes after varying schedules of 5-fluorouracil in rats. J. Pharmacol. Exp. Ther. 2000, 295 (2), 734-740.

13. Minami, H.; Sasaki, Y.; Saijo, N.; Ohtsu, T.; Fujii, H.; Igarashi, T.; Itoh, K. Indirect-response model for the time course of leukopenia with anti-cancer drugs. Clin. Pharmacol. Ther. 1998, 64, 511-521.

14. Bugat, R.; Canal, P.; Chatelut, E. Population pharmacokinetics of topotecan: Intraindividual variability of total drug. Cancer Chemother. Pharmacol. 2000, 46 (5), 375381.

15. O'Reilly, S. Topotecan: What dose, what schedule, what route? Clin. Cancer Res. 1999, 5, 3-5. 


\section{Request Permission or Order Reprints Instantly!}

Interested in copying and sharing this article? In most cases, U.S. Copyright Law requires that you get permission from the article's rightsholder before using copyrighted content.

All information and materials found in this article, including but not limited to text, trademarks, patents, logos, graphics and images (the "Materials"), are the copyrighted works and other forms of intellectual property of Marcel Dekker, Inc., or its licensors. All rights not expressly granted are reserved.

Get permission to lawfully reproduce and distribute the Materials or order reprints quickly and painlessly. Simply click on the "Request Permission/ Order Reprints" link below and follow the instructions. Visit the U.S. Copyright Office for information on Fair Use limitations of U.S. copyright law. Please refer to The Association of American Publishers' (AAP) website for guidelines on Fair Use in the Classroom.

The Materials are for your personal use only and cannot be reformatted, reposted, resold or distributed by electronic means or otherwise without permission from Marcel Dekker, Inc. Marcel Dekker, Inc. grants you the limited right to display the Materials only on your personal computer or personal wireless device, and to copy and download single copies of such Materials provided that any copyright, trademark or other notice appearing on such Materials is also retained by, displayed, copied or downloaded as part of the Materials and is not removed or obscured, and provided you do not edit, modify, alter or enhance the Materials. Please refer to our Website User Agreement for more details.

\section{Request Permission/Order Reprints}

Reprints of this article can also be ordered at http://www.dekker.com/servlet/product/DOI/101081EEBS120023192 